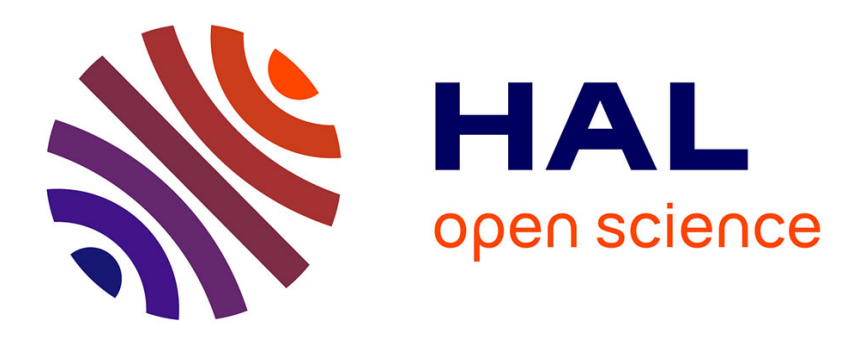

\title{
Frame-based Gaussian beam shooting from experimental far field data
}

\author{
Igor Francisco Arias Lopez, Christine Letrou
}

\section{To cite this version:}

Igor Francisco Arias Lopez, Christine Letrou. Frame-based Gaussian beam shooting from experimental far field data. ICEAA 2014: International Conference on Electromagnetics in Advanced Applications, Aug 2014, Palm Beach, Aruba. pp.532 - 535, 10.1109/ICEAA.2014.6903914 • hal-01262400

\section{HAL Id: hal-01262400 \\ https://hal.science/hal-01262400}

Submitted on 26 Jan 2016

HAL is a multi-disciplinary open access archive for the deposit and dissemination of scientific research documents, whether they are published or not. The documents may come from teaching and research institutions in France or abroad, or from public or private research centers.
L'archive ouverte pluridisciplinaire HAL, est destinée au dépôt et à la diffusion de documents scientifiques de niveau recherche, publiés ou non, émanant des établissements d'enseignement et de recherche français ou étrangers, des laboratoires publics ou privés. 


\section{Frame-based Gaussian beam shooting from experimental far field data}

\author{
I.F. Arias Lopez*
}

\begin{abstract}
Frame decomposition is conventionally used to perform decomposition of fields radiated by large planar apertures into a half plane, in a rigorous and stable way. An algorithm based on a spectral partitioning concept has been developed to extend frame-based Gaussian beam launching to omnidirectional and non directive sources. Frame decomposition is applied in six planes in the spectral domain, starting from the knowledge of the radiated far field, which is partitioned through a "partition of unity" procedure. This communication outlines this method, then focuses on its application and test in a real life contextwhere initial far field data are obtained from measurements.
\end{abstract}

\section{INTRODUCTION}

Frame theory provides a rigorous framework to represent source field distributions in the form of superpositions of translated and phase shifted Gaussian windows $[1,2]$. These Gaussian windows radiate fields in the form of paraxial Gaussian beams, in as much as their spectrum is sufficiently localized. Frame decomposition has thus been introduced as the initial discretization step in some Gaussian beam shooting (GBS) algorithms [3, 4]. It was also introduced to re-expand diffracted fields on planar obstacles or apertures, through a re-expansion algorithm performed in the course of Gaussian beam tracking in multi-reflecting environments [5].

Until recently, only planar source distributions were amenable to frame decomposition, and frame based GBS was better suited to highly directive radiating apertures than to less directive ones, due to inaccuraccies occuring in paraxial beam expressions for highly tilted beams.

In order to generalize frame-based Gaussian beam shooting to a wider range of source distributions and of antennas, with preserved accuracy and rigor, we have developed an algorithm based on a $3 \mathrm{~d}$ spectral domain partition.

Given an antenna far field, its plane wave spectrum (PWS) is known in any plane, hence in six planes, taken parallel to cube faces, radiating into

\footnotetext{
* CENDIT Foundation, Dirección de Propagacion y Antenas, Base Aerea Generalisimo Francisco de Miranda, Complejo Tecnologico Simón Rodriguez, Caracas,Venezuela, email: igor_arias2004@yahoo.com, tel.: +58 4127065773.

†Télécom SudParis (Lab. SAMOVAR - UMR CNRS 5157), 9 rue Charles Fourier, 91011 Evry Cedex, France
}

six half-spaces. A partition of unity is then formulated which allows to synthetize the $3 \mathrm{~d}$ far field from the summation of fields radiated by these six spectra, each multiplied by a partitioning window. Frame decomposition is then applied to these "partial spectra", in each of the six planes, enabling GBS from all the planes. Fields radiated by the six partial PWS are summed to obtain the $3 \mathrm{~d}$ radiated fields.

Section 2 gives an outline of the "spectral partitioning" formulation. Section 3 presents its application to experimental horn fields, the initial far field data being obtained from measurements.

\section{FORMULATION}

In this section, frame decomposition is first presented as applied to functions of one variable, to introduce frame parameters and frame theory concepts, then to functions of two variables, in order to discretize field components of radiating planar distributions. Finally, six decompositions in six different planes are combined in order to discretize fields radiated by any type of source.

Harmonic time dependence $e^{-i \omega t}$, with $\omega$ the angular frequency, is assumed and suppressed in equations. The Fourier transform of a function $g \in L^{2}(\mathbb{R})$, denoted $\widetilde{g}$, is defined as:

$$
\widetilde{g}\left(k_{x}\right)=\int_{-\infty}^{+\infty} g(x) e^{-i k_{x} x} d x
$$

\subsection{Frame based Gaussian beam shooting}

In the $L^{2}(\mathbb{R})$ Hilbert space, the set of Gaussian functions

$$
\begin{gathered}
w_{m n}(x)=w(x-m \bar{x}) e^{i n \bar{k}_{x}(x-m \bar{x})},(m, n) \in \mathbb{Z}^{2} \\
\text { with } \quad w(x)=\sqrt{\frac{\sqrt{2}}{L}} e^{-\pi \frac{x^{2}}{L^{2}}}
\end{gathered}
$$

is a frame if and only if $\bar{x} \bar{k}_{x}=2 \pi \nu$ with $\nu<1$ (oversampling factor) [1]. $\bar{x}$ and $\bar{k}_{x}$ are respectively the spatial and spectral domain translation steps.

Frames are complete sets hence any function $f \in L^{2}(\mathbb{R})$ can be expressed as a weighted summation of the frame windows:

$$
f=\sum_{(m, n) \in \mathbb{Z}^{2}} a_{m n} w_{m n}
$$


with the (non unique) $a_{m n}$ complex coefficients called "frame coefficients". These coefficients can be calculated by projecting the function on a "dual frame" of functions $[2,4]$.

By Fourier transform of equation (1), the decomposition of $\tilde{f}$ on the frame obtained by translating the Gaussian window $\widetilde{w}$ is easily obtained as:

$$
\widetilde{f}=\sum_{(m, n) \in \mathbb{Z}^{2}} a_{m n} e^{i m n \bar{k}_{x} \bar{x}} \widetilde{w}_{m n}
$$

As is visible from equations (1) and (2), frame coefficients can be calculated either in the initial domain or in the transformed one.

Frames in $L^{2}\left(\mathbb{R}^{2}\right)$ are defined as product frames: $w_{m n p q}=w_{m n} w_{p q},(m, n, p, q) \in \mathbb{Z}^{4}$. If a $y$ polarized field distribution radiating into the $z>0$ half-space is decomposed on such a frame, with $A_{m n p q}$ the frame coefficients, then the radiated field at any point $M$ with $z>0$ is obtained as:

$$
\vec{E}(M)=\sum_{m, n, p, q} A_{m n p q} e^{i\left(m n l_{x} \kappa_{x}+p q l_{y} \kappa_{y}\right)} \vec{B}_{m n p q}(M)
$$

where $\vec{B}_{m n p q}$ is the field radiated by the plane wave spectrum (PWS) associated to the $w_{m n p q}$ frame window used to discretize the $E_{y}(x, y)$ function. For spectrally narrow frame windows, the $\vec{B}_{m n p q}$ radiated field can be expressed in the form of a Gaussian beam (ray type expression with complex curvature), based on a paraxial approximation in the spectral domain $[3,4]$.

\subsection{Spectral partitioning}

In this subsection, partial PWS are defined in six different planes, by multiplying the PWS of the antenna in each plane by a "partitioning function". The partitioning functions are introduced to obtain the field at any observation point outside of the reactive region of the radiating source by summing up the fields calculated by GBS from several of these planes, based on frame decomposition of the partial PWS.

\subsubsection{Notations}

The far field radiated by the antenna is assumed to be known in all $(\theta, \phi)$ directions in the global coordinate system $(O, \hat{x}, \hat{y}, \hat{z})$. Six coordinate systems $\mathcal{S}_{j}=\left(O, \hat{x}_{j}, \hat{y}_{j}, \hat{z}_{j}\right), j=1, \ldots, 6$, are introduced, and plane wave spectra are defined in the six $z_{j}=0$ planes, called $\mathcal{P}_{j}=\left(O, \hat{x}_{j}, \hat{y}_{j}\right)$. Figure 1 gives an exploded view of the $\mathcal{P}_{j}$ planes and of their associated spectral variables $\left(k_{x_{j}}, k_{y_{j}}\right)$. The PWS of the

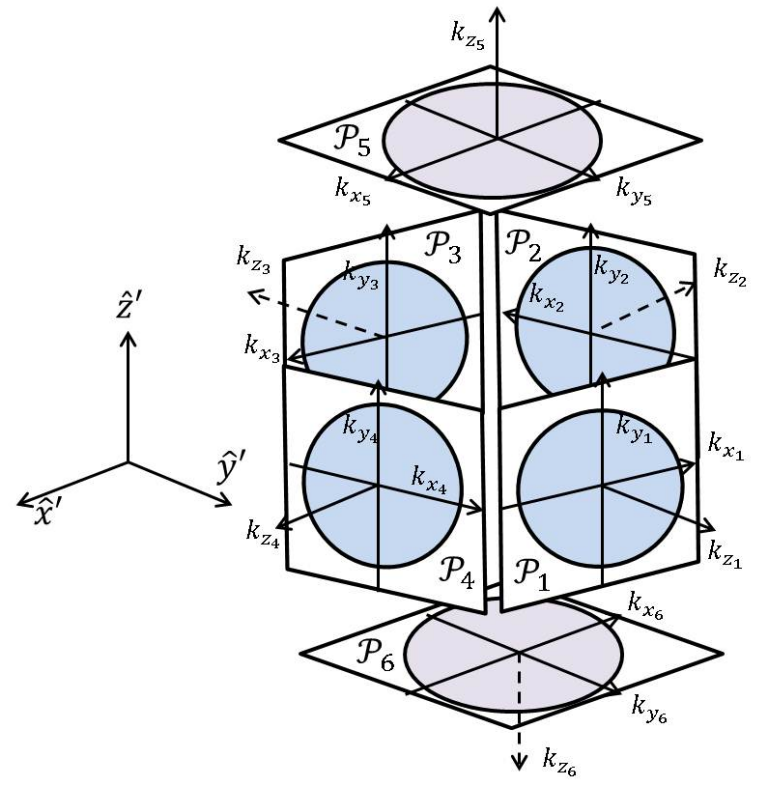

Figure 1: $\mathcal{P}_{j}$ planes and associated spectral variables.

antenna in the $\mathcal{P}_{j}$ plane is denoted $\overrightarrow{\tilde{E}}^{(j)}\left(k_{x_{j}}, k_{y_{j}}\right)$ and radiates into the $z_{j}>0$ half-space, noted $\mathcal{H}_{j}$.

Any point $M$ in the $3 \mathrm{~d}$ space, except for the origin $O$ of all $\mathcal{P}_{j}$ planes, belongs to exactly three different $\mathcal{H}_{j}, j \in J$ half-spaces.

\subsubsection{Partition of unity relation}

The classical asymptotic expression of the radiated far field in a given half-space as a function of a PWS radiating into this half-space, makes it easy to deduce each $\overrightarrow{\tilde{E}}^{(j)}$ PWS from the antenna far field in the $\mathcal{H}_{j}$ half-space.

Partial plane wave spectra (after partitioning) are defined in the $\mathcal{P}_{j}$ planes as:

$$
\overrightarrow{\tilde{E}}^{\chi_{j}}\left(k_{x_{j}}, k_{y_{j}}\right)=\overrightarrow{\vec{E}}^{(j)}\left(k_{x_{j}}, k_{y_{j}}\right) \chi_{j}\left(k_{x_{j}}, k_{y_{j}}\right)
$$

with $\chi_{j}\left(k_{x_{j}}, k_{y_{j}}\right), j=1, \ldots, 6$ partitioning functions.

In order to obtain the radiated far field of the antenna by summing up the fields radiated by all these partial PWS, the partitioning functions must verify $[6]$ :

$$
\sum_{j \in J} \chi_{j}\left(k_{x_{j}}, k_{y_{j}}\right)=1
$$

for any direction in the $3 \mathrm{~d}$ space, defined by a wavevector $\vec{k} . \quad J$ is the set of indices such that $k_{z_{j}}>0$, and $\left(k_{x_{j}}, k_{y_{j}}\right)$ are the components of $\vec{k}$ along the $\hat{x}_{j}$ and $\hat{y}_{j}$ vectors. 


\subsubsection{Partition of unity functions}

The two variable partitioning functions are obtained:

- as the product of two one-variable partitioning functions of variables $\left(k_{x_{j}}\right.$ and $\left.k_{y_{j}}\right)$, in planes $\mathcal{P}_{j}, j=1, \ldots, 4$ ("lateral" planes);

- as a one-variable partitioning function of $k_{r_{j}}=$ $\sqrt{k_{x_{j}}^{2}+k_{y_{j}}^{2}}$, in planes $\mathcal{P}_{j}, j \in\{5,6\}$ (planes oriented toward "bottom" and "top").

With this choice, the $\chi_{5}$ and $\chi_{6}$ functions are rotationally symmetric around the $z^{\prime}$ axis. We also impose that $\chi_{1}=\chi_{3}$ and $\chi_{2}=\chi_{4}$.

The one-variable partitioning functions are derived from the Hann window, in order to minimze the widening effect in space of spectral truncation [7]. Figures 2 and 3 present the $\chi_{1}$ and $\chi_{5}$ functions, respectively.

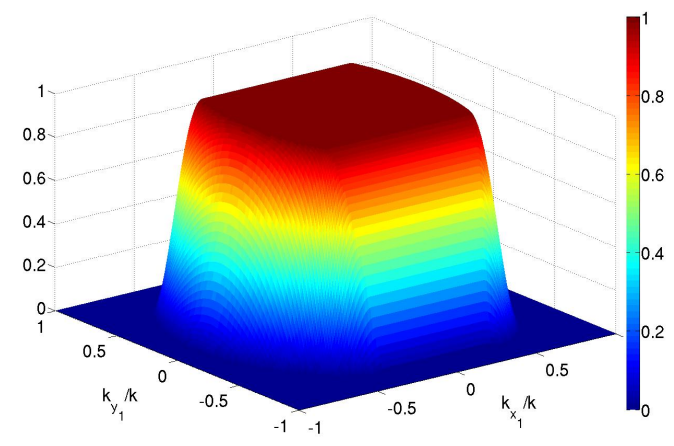

Figure 2: Two-variable $\chi_{1}$ partitioning function.

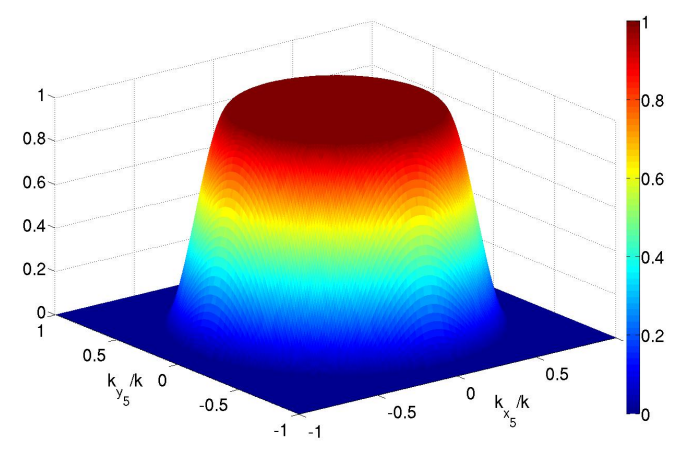

Figure 3: Two-variable $\chi_{5}$ partitioning function.

\section{NUMERICAL RESULTS}

The algorithm presented in the previous section has been validated in the case of analytically defined omnidirectional radiation patterns (halfwavelength dipole, dipole array). The far field of such antennas is synthetized by GBS from 6 planes where spectral partitioning has been applied to the initially known antenna PWS [6].

In real life contexts, antennas are generally known by their far field radiation patterns, and more accurate simulations (of propagation for instance) are obtained if measured antenna patterns are used to characterize the antenna radiation, instead of closed form theoretical expressions. We have applied the frame based GBS algorithm including spectral partitioning to the measured far field of a horn.

Frame coefficient computation involves a discrete Fourier transform in the spectral domain, and the required data points are on a regular grid in each of the six planes used for GBS, but by no means on a regular grid on a sphere centered on the antenna. For this reason, the measured far field data need to be interpolated in the course of frame coefficient calculation.

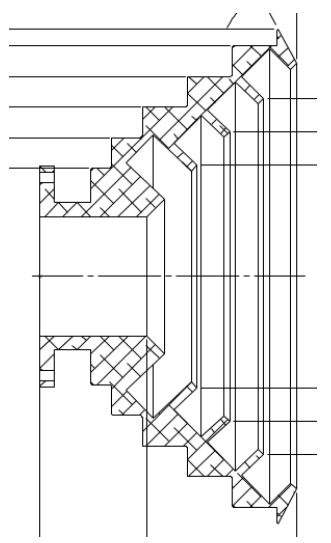

Figure 4: Corrugated horn (cut).

The measured horn is a corrugated horn shown in Figure 4. Its aperture diameter is 24 centimeters, and it was tested at $6 \mathrm{GHz}$ on a sphere of radius 5 meters, in the far field of the antenna, with a 2 degree step along $\phi$ and $\theta$. A rectangular horn is used as testing antenna. Co- and crosspolarizations (linear ones) patterns were measured.

Figures 5 and 6 present the measured and synthetized far field of this horn, which compare well. Figures 7 and 8 present a comparison of $1 \mathrm{~d}$ patterns, for one cartesian component in the far field. Except for inaccuracies in measured data (alignment), the two patterns compare very well again. 


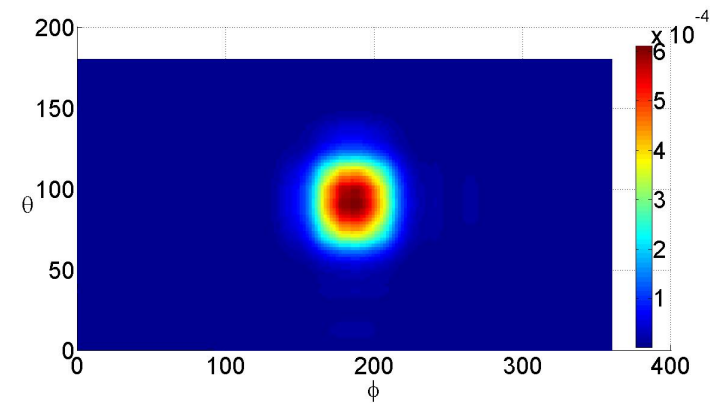

Figure 5: Horn measured far field magnitude.

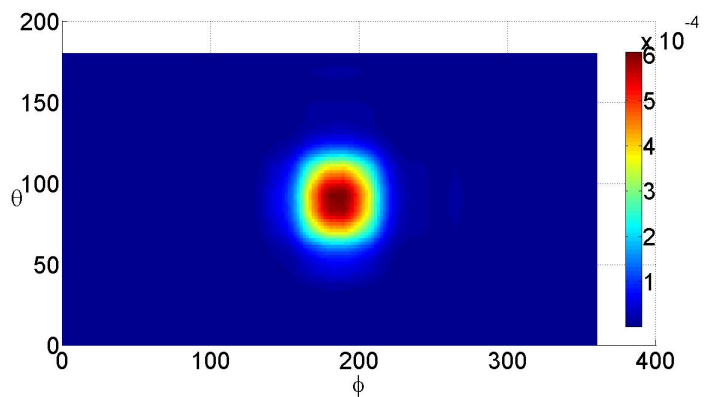

Figure 6: Horn far field magnitude synthetized by GBS.

\section{CONCLUSION}

The "spectral partitioning" algorithm has been shown to efficiently complement the usual framebased Gaussian beam shooting method, in the case of non directive antenna fields. The method has been applied to experimentally known patterns (discretized measured data). The accuracy of the fields computed by GBS is assessed by comparison with the initial measured data in the far field. Results assessing the ability of the method to perform far to "near" (intermediate zone) field transformation will be presented at the conference.

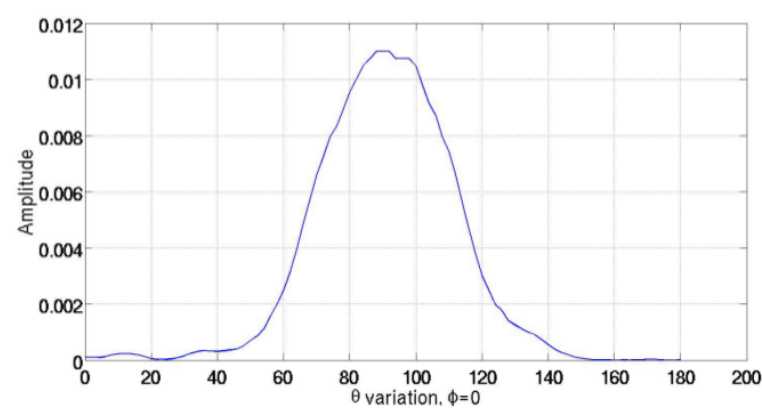

Figure 7: Horn measured far field component.

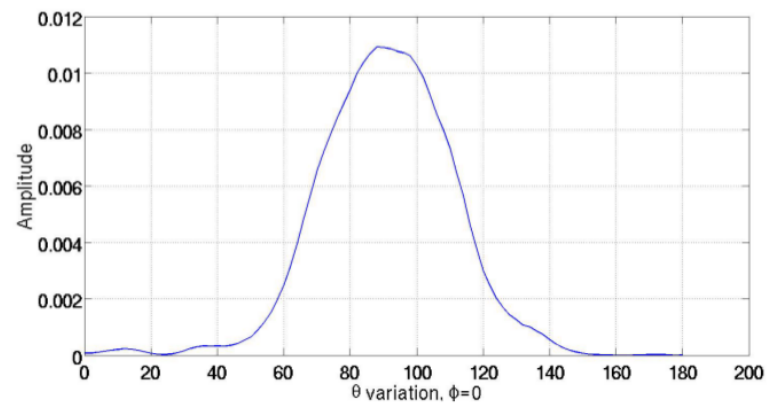

Figure 8: Horn far field component synthetized by GBS.

\section{References}

[1] D. Lugara and C. Letrou, "Alternative to Gabor's representation of plane aperture radiation," Electron. Lett., vol. 34, no. 24, pp. 22862287, Nov. 1998.

[2] — , "Printed antennas analysis by a Gabor frame-based method of moments," IEEE Trans. Antennas Propagat., vol. 50, no. 11, pp. 15881597, 2002.

[3] D. Lugara, C. Letrou, A. Shlivinski, E. Heyman, and A. Boag, "Frame-based Gaussian beam summation method: Theory and application," Radio Science, vol. 38, no. 2, Apr. 2003.

[4] A. Shlivinski, E. Heyman, A. Boag, and C. Letrou, "A phase-space beam summation formulation for ultrawide-band radiation," IEEE Trans. Antennas Propagat., vol. 52, no. 8, pp. 2042-2056, 2004.

[5] I. Ghannoum, C. Letrou, and G. Beauquet, "Frame based Gaussian beam bouncing," in EMTS'10 : 20th International Symposium on Electromagnetic Theory, IEEE, Ed., 2010, pp. $68-71$.

[6] I. A. Lopez and C. Letrou, "Gaussian beam launching based on frame decomposition and $3 \mathrm{~d}$ spectral partition," in ICEAA '13 : Int. Conf. on Electromagnetics in Advanced Applications, Torino, Italy, Sept. 2013, pp. $692-695$.

[7] - "Frame decomposition of scattered fields," in ICEAA '11 : Int. Conf. on Electromagnetics in Advanced Applications, Torino, Italy, Sept. 2011, pp. $1261-1264$. 